\title{
A Time-Stepping DRBEM for 3D Anisotropic Functionally Graded Piezoelectric Structures Under the Influence of Gravitational Waves
}

\author{
Mohamed Abdelsabour Fahmy ${ }^{1,2(\bowtie)}$ \\ ${ }^{1}$ Faculty of Computers and Informatics, Suez Canal University, \\ New Campus, 4.5 Km, Ring Road, El Salam District, Ismailia 41522, Egypt \\ mohamed_fahmy@ci.suez.edu.eg, maselim@uqu.edu.sa \\ 2 Jummum University College, Umm Al-Qura University, \\ Jummum, Mecca 21955, Saudi Arabia
}

\begin{abstract}
A three-dimensional time-stepping dual reciprocity boundary element method (DRBEM) modelling was investigated for anisotropic functionally graded piezoelectric Structure (FGPS) under the influence of gravitational waves. The FGPS is assumed to be graded through the thickness. The basic aim of this paper is to describe anisotropic displacement behaviour of the homogeneous piezoelectric structures (HPS) and FGPS under the influence of Gravitational waves. The accuracy of the proposed method was examined and confirmed by comparing the obtained results with those known previously. Understanding the behaviour of anisotropic FGPS under the effect of gravitational waves is the key to extending the application of these structures.
\end{abstract}

\begin{tabular}{ll}
\multicolumn{2}{l}{ Nomenclature } \\
$x$ & Field point \\
$\xi$ & Load point \\
$\mathrm{t}$ & Time \\
$r$ & Euclidean distance \\
$m$ & Dimensionless constant \\
$\mathrm{G}$ & Gravitational acceleration \\
$\rho$ & Density \\
$\Phi$ & Electric potential \\
$u_{h}$ & Displacement components \\
$\sigma_{\mathrm{fg}}$ & Mechanical stress tensor \\
$\varepsilon_{\mathrm{hi}}$ & Mechanical strain tensor \\
$\mathrm{E}_{\mathrm{i}}$ & Electric field vector \\
$\mathrm{C}_{\mathrm{fghi}}$ & Elasticity tensor \\
$\mathrm{e}_{\mathrm{fhi}}$ & Piezoelectric tensor \\
$\epsilon_{\mathrm{fi}}$ & Permittivity tensor \\
$\mathrm{D}$ & Electric displacement
\end{tabular}




\section{Introduction}

Functionally graded piezoelectric structures (FGPSs) considered in the current study have been received much attention in recent years due to their various applications including sensors and actuators, aerospace, nuclear energy, chemical plant, electronics, biomaterials, piezoelectric motors, reduction of vibrations and noise, infertility treatment, ultrasonic micromotors, micropumps, microvalves and photovoltaics. Since it is very difficult to find the analytical solution to the considered problem, therefore, an important number of engineering and mathematical papers devoted to the numerical solution have been studied to describe the global behavior of such problems (Suo et al. 1992; Pan 1999; Jin and Zhong 2002; Zhang et al. 2002; Lin et al. 2003; Fang et al. 2004; Abd-Alla and Al-sheikh 2009b; Kuna 2010; Davì and Milazzo 2011; Abd-Alla et al. 2012 and Alibeigloo and Liew 2015).

The advantages in the boundary element method (BEM) arises from the fact that the BEM can be regarded as boundary-based method that uses the boundary integral equation formulations where only the boundary of the domain of the partial differential equation (PDE) is required to be meshed. But in the domain-based methods such the finite element method (FEM), finite difference method (FDM) and element free method (EFM) that use ordinary differential equation (ODE) or PDE formulations, where the whole domain of the PDE requires discretisation. Thus the dimension of the problem is effectively reduced by one, that is, surfaces for three-dimensional (3D) problems or curves for two-dimensional (2D) problems. And the equation governing the infinite domain is reduced to an equation over the finite boundary. Also, the BEM can be applied along with the other domain-based methods to verify the solutions to the problems that do not have available analytical solutions. Presence of domain integrals in the formulation of the BEM dramatically decreases the efficiency of this technique. One of the most frequently used techniques for converting the domain integral into a boundary one is the so-called dual reciprocity boundary element method (DRBEM). This method was initially developed by Nardini and Brebbia (1983) in the context of two-dimensional (2D) elastodynamics and has been extended to deal with a variety of problems wherein the domain integral may account for linear-nonlinear static-dynamic effects. A more extensive historical review and applications of dual reciprocity boundary element method may be found in (Wrobel and Brebbia 1987; Partridge and Brebbia 1990; Partridge and Wrobel 1990; Frijns et al. 2000; Gaul 2003 and Fahmy Fahmy 2011, 2012a, b, c, d, e, 2013a).

In this paper the governing equations of an anisotropic FGPS under the influence of gravity are solved by means of a time-stepping dual reciprocity boundary element method (DRBEM) to describe the displacement behavior of the homogeneous and functionally graded structures under the influence of gravity. The accuracy of the proposed method was examined and confirmed by comparing the obtained results with those known previously. 


\section{Formulation of the Problem}

Consider a Cartesian coordinates system Oxyz as shown in Fig. 1. We shall consider an anisotropic functionally graded piezoelectric structure under the influence of gravity. The plate occupies the region $R=\{(x, y, z): 0<x<\underline{\gamma}, 0<y<\underline{\beta}, 0<z<\underline{\alpha}\}$ with graded material properties in the thickness direction.

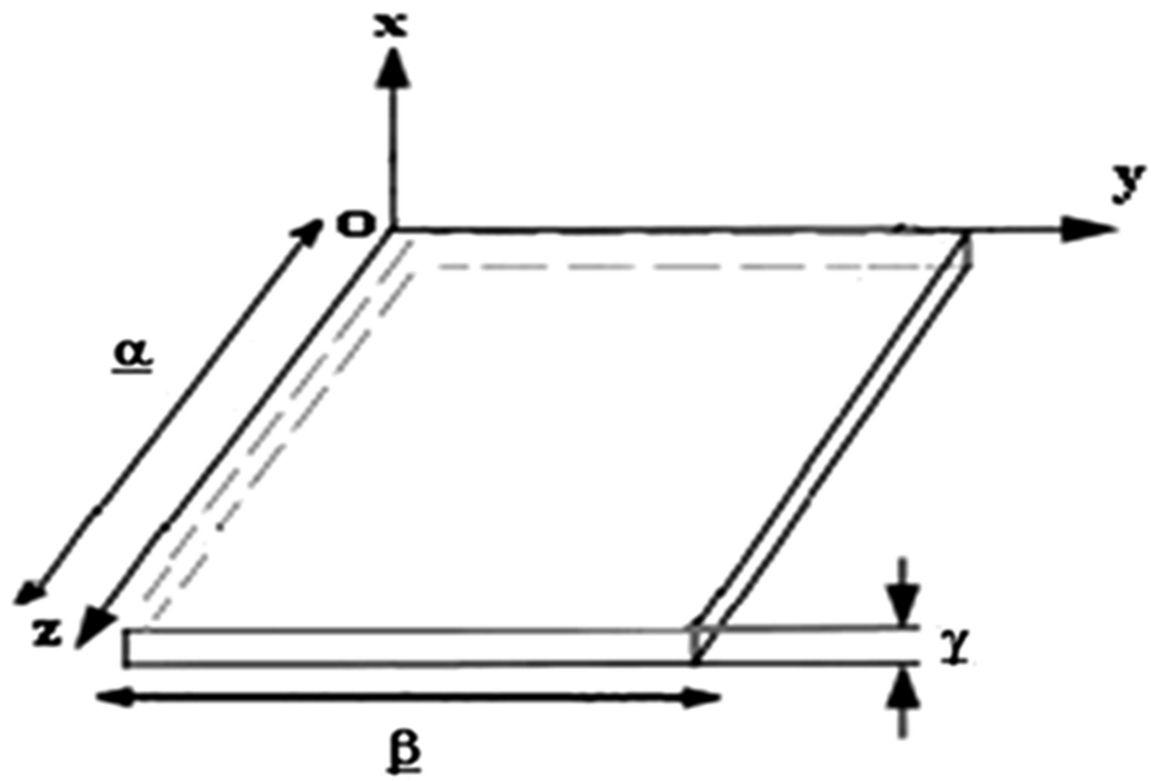

Fig. 1. The coordinate system of the FGPS.

The governing equations for anisotropic functionally graded piezoelectric plate under the influence of gravity may be written in the following form

$$
\begin{gathered}
\sigma_{f g, f}=\rho(x+1)^{m} \ddot{u}_{g}+\rho g(x+1)^{m}\left(u_{g, f}+u_{f, g}\right) \\
D_{f, f}=0
\end{gathered}
$$

In which

$$
\begin{gathered}
\sigma_{f g}=(x+1)^{m}\left[C_{f g h i} \varepsilon_{h i}-e_{i f g} E_{i}\right] \\
D_{f}=(x+1)^{m}\left[e_{f h i} \varepsilon_{h i}+\epsilon_{f i} E_{i}\right]
\end{gathered}
$$

where 


$$
\begin{gathered}
\varepsilon_{h i}=\frac{1}{2}\left(u_{h, i}+u_{i, h}\right) \\
E_{i}=-\Phi_{, i}
\end{gathered}
$$

By substituting of Eqs. (3)-(6) into Eqs. (1) and (2) we obtain

$$
\begin{gathered}
{\left[C_{f g h i} u_{h, i f}+e_{i f g} \Phi_{, i f}\right]+\frac{m}{x+1}\left[C_{f g h i} u_{h, i}+e_{i f g} \Phi_{, i}\right]} \\
=\rho \ddot{u}_{g}+\rho g(x+1)^{m}\left(u_{g, f}+u_{f, g}\right) \quad\left(C_{f g h i}=C_{g f h i}=C_{h i f g}\right) \\
{\left[e_{f h i} u_{h, i f}-\epsilon_{f i} \Phi_{, i f}\right]+\frac{m}{x+1}\left[e_{f h i} u_{h, i}-\epsilon_{f i} \Phi_{, i}\right]=0 \quad\left(e_{f h i}=e_{f i h}, \epsilon_{f i}=\epsilon_{i f}\right)}
\end{gathered}
$$

A superposed dot denotes differentiation with respect to the time and a comma followed by a subscript denotes partial differentiation with respect to the corresponding coordinates.

Now the governing Eqs. (7) and (8) may be rewritten in the following form

$$
\begin{gathered}
C_{f g h i} u_{h, i f}=\rho \ddot{u}_{g}+\rho g(x+1)^{m}\left(u_{g, f}+u_{f, g}\right)-C_{f g h i} \aleph u_{h, i}-e_{i f g}\left\lfloor\Phi_{, i f}+\aleph \Phi_{, i}\right\rfloor \\
e_{f h i} u_{h, i f}=\epsilon_{f i} \Phi_{, i f}-e_{f h i} \aleph u_{h, i}+\epsilon_{f i}\left[\Phi_{, i f}+\aleph \Phi_{, i}\right]
\end{gathered}
$$

where $\aleph=\frac{m}{x+1}$

\section{Numerical Implementation}

The governing Eqs. (9) and (10) can be reformulated in terms of operators as follows

$$
\begin{aligned}
& L_{f g} u_{h}=b_{f g} \\
& L_{f h} \Phi=b_{f h}
\end{aligned}
$$

Where

$$
\begin{gathered}
L_{f g}=C_{f g h i} \frac{\partial}{x_{i}} \frac{\partial}{x_{f}} \\
b_{f g}=\rho \ddot{u}_{g}+\rho g(x+1)^{m}\left(u_{g, f}+u_{f, g}\right)-C_{f g h i} \aleph u_{h, i}-e_{i f g}\left\lfloor\Phi_{, i f}+\aleph \Phi_{, i}\right\rfloor \\
L_{f h}=e_{f h i} \frac{\partial}{x_{i}} \frac{\partial}{x_{f}} \\
b_{f h}=\epsilon_{f i} \Phi_{, i f}-e_{f h i} \aleph u_{h, i}+\epsilon_{f i}\left[\Phi_{, i f}+\aleph \Phi_{, i}\right]
\end{gathered}
$$


It is useful to write the governing equations in contracted notation form, we have used the following contracted notation to introduce generalized piezoelectric vectors and tensors, which contain corresponding elastic and electric variables as follows:

$$
\begin{gathered}
U_{H}= \begin{cases}u_{h} & h=H=1,2,3 \\
\Phi & H=4\end{cases} \\
\mathrm{Z}_{G}= \begin{cases}t_{g} & g=G=1,2,3 \\
q & G=4\end{cases} \\
C_{f G H i}=\left\{\begin{array}{lll}
C_{f g h i}, & g=G=1,2,3 ; & h=H=1,2,3 \\
e_{i f g}, & g=G=1,2,3 ; & H=4 \\
e_{f h i}, & G=4 ; & h=H=1,2,3 \\
-\epsilon_{f i}, & G=4 ; & H=4
\end{array}\right.
\end{gathered}
$$

Using the following Kronecker delta form

$$
\delta_{G H}= \begin{cases}\delta_{g h} & g=G=1,2,3, \quad k=K=1,2,3, \\ 0 & \text { otherwise }\end{cases}
$$

The governing equations can be combined to yield

$$
L_{G H} U_{H}=\rho \delta_{G H} \ddot{U}_{H}-B_{G}
$$

where

$$
\begin{aligned}
L_{G H} & =C_{f G H i} \frac{\partial}{x_{i}} \frac{\partial}{x_{f}} \\
B_{G} & = \begin{cases}-\rho \ddot{u}_{g}+\rho g(x+1)^{m}\left(u_{g, f}+u_{f, g}\right)+C_{f g h} \overline{\bar{u}}+e_{i f g} \overline{\bar{\Phi}}, & g=G=1,2,3 \\
\epsilon_{f i} \Phi_{, i f}-e_{f h i} \overline{\bar{u}}+\epsilon_{f i} \overline{\bar{\Phi}}, & G=4\end{cases} \\
\overline{\bar{u}} & =\aleph u_{h, i}, \quad \overline{\bar{\Phi}}=\Phi_{, i f}+\aleph \Phi_{, i}
\end{aligned}
$$

Now, we choose the fundamental solution $\mathrm{U}_{\mathrm{MH}}^{*}$ as weighting function as follows

$$
L_{G H} U_{M H}^{*}(x, \xi)=-\delta_{G M} \delta(x, \xi)
$$

The weighted residual formula is integrated by parts twice to obtain the following piezoelectric reciprocity relation

$$
\int_{R}\left(L_{G H} U_{H} U_{M G}^{*}-L_{G H} U_{M H}^{*} U_{G}\right) d R=\int_{\Gamma}\left(U_{M G}^{*} \mathrm{Z}_{G}-\mathrm{Z}_{M G}^{*} U_{G}\right) d \Gamma
$$

where 


$$
\mathrm{Z}_{M G}^{*}=C_{f G H i} U_{M H, i}^{*} n_{f}
$$

Making use of the sifting property, we obtain from Eq. (23) the piezoelectric representation formula

$$
U_{M}(\xi)=\int_{\Gamma}\left(U_{M G}^{*} \mathrm{Z}_{G}-\mathrm{Z}_{M G}^{*} U_{G}\right) d \Gamma-\int_{R} U_{M G}^{*}\left(\rho \delta_{G H} \ddot{U}_{H}-B_{G}\right) d R
$$

The DRBEM is employed in Eq. (24) to transform the domain integrals into boundary integrals, hence we may deduce the following piezoelectric dual reciprocity representation formula

$$
\begin{aligned}
U_{H}(\xi) & =\int_{\Gamma}\left(U_{H G}^{*} \mathrm{Z}_{G}-\mathrm{Z}_{H G}^{*} U_{G}\right) d \Gamma \\
& +\sum_{q=1}^{N}\left(U_{H N}^{q}(\xi)+\int_{\Gamma}\left(T_{H G}^{*} U_{G N}^{q}-U_{H G}^{*} T_{G N}^{q}\right) d \Gamma\right) \alpha_{N}^{q}
\end{aligned}
$$

Now the source term in Eq. (24) is approximated by a series of tensor functions $\mathrm{f}_{\mathrm{GN}}^{\mathrm{q}}$ and unknown coefficients $\alpha_{\mathrm{N}}^{\mathrm{q}}$ as follows

$$
\rho \delta_{G H} \ddot{U}_{H}-B_{G} \approx \sum_{q=1}^{N} f_{G N}^{q} \alpha_{N}^{q}
$$

Using the n-dimensional thin plate splines (TPS), the Green's function is chosen to be of the form $G(x, s)=u(|x-s|), x \in R^{n}, s \in R^{n}$. The function $u(r)$ is a solution to the equation

$$
\begin{aligned}
\delta(r) & =\Delta^{2} u(r) \\
& =u^{(4)}(r)+\frac{2(n-1)}{r} u^{(3)}(r)+\frac{(n-1)(n-3)}{r^{2}} u^{(2)}(r)-\frac{(n-1)(n-3)}{r^{3}} u^{(1)}(r)
\end{aligned}
$$

For $r \in\left[0, \infty\left[\right.\right.$, where $u^{(k)}(r)$ is the $k-t h$ order derivative of $u(r), r=\|x-\xi\|$ is the Euclidean distance between the field point $x$ and the load point $\xi$.

$$
U_{G N}^{q}= \begin{cases}C_{0}+C_{1} r^{2}+C_{2} \ln r+C_{3} r^{2} \ln r, & n=2 \\ C_{0}+C_{1} r^{2}+C_{2} \ln r+C_{3} r^{-2}, & n=4 \\ C_{0}+C_{1} r^{2}+C_{2} r^{2-n}+C_{3} r^{4-n}, & \text { all other } n\end{cases}
$$

Hence, the traction particular solution $T_{G N}^{q}$ and the source function $f_{G N}^{\mathrm{q}}$ can be obtained by evaluating

$$
T_{G N}^{q}=C_{f G H i} U_{H N, i}^{q} n_{f}, L_{G H} U_{H N}^{q}=f_{G N}^{q}
$$


According to Fahmy (2013b, c, 2014a, b), the dual reciprocity boundary integral Eq. (25) can be written in the following system of equations

$$
\zeta \check{U}(t)-\eta \check{T}(t)=(\zeta \check{\zeta}(t)-\eta \check{\tau}(t)) \alpha(t)
$$

where $\zeta, \eta$ are BEM system matrices, $\check{U}, \check{T}$ contain the nodal values of the generalized displacements and fluxes, and $\check{\mho}, \check{\tau}$ contain the particular solutions

$$
\begin{gathered}
{\left[\begin{array}{c}
\rho \check{\ddot{u}}-\rho \check{b} \\
0
\end{array}\right]=\left[\begin{array}{ll}
F_{11} & F_{14} \\
F_{41} & F_{44}
\end{array}\right]\left[\begin{array}{l}
\alpha_{1} \\
\alpha_{4}
\end{array}\right]} \\
C_{H G}(\xi) U_{G}(\xi)+C P V \int_{\Gamma} \mathrm{Z}_{H G}^{*} U_{G} d \Gamma=\int_{\Gamma} U_{H G}^{*} \mathrm{Z}_{G} d \Gamma
\end{gathered}
$$

The coefficient vector $\alpha_{s}(t)$ can be calculated by setting up a system of $N$ equations from (26) using the point collocation procedure, which yield

$$
\rho \check{\ddot{u}}-\rho \hat{b}=\mathcal{F}_{11} \alpha_{\mathrm{s}}(\mathrm{t})
$$

where

$$
\begin{gathered}
\mathcal{F}_{11}=F_{11}-F_{14} F_{44}^{-1} F_{41} \\
\alpha_{s}(t)=\mathcal{F}^{-1}(\rho \check{\ddot{U}}(t)-\check{B}(t)) \\
\mathcal{F}^{-1}= \begin{cases}\mathcal{F}_{11}^{-1} & s=1 \\
-\mathcal{F}_{44}^{-1} F_{41} \mathcal{F}_{11}^{-1} & s=4 \\
0 & \text { otherwise }\end{cases}
\end{gathered}
$$

Substitution of (34) into (29) yields the system

$$
M \check{\ddot{U}}(t)+\zeta \check{U}(t)=\eta \check{T}(t)+\check{\mathcal{B}}(t)
$$

where the volume matrix $\mathrm{V}$, piezoelectric mass matrix $\mathrm{M}$ and source vector $\check{\mathcal{B}}(t)$ are as follows:

$$
V=(\eta \check{\tau} c(t)-\zeta \check{\zeta}(t)) \mathcal{F}^{-1}, \quad M=\rho V, \quad \check{\mathcal{B}}(t)=V \check{B}(t)
$$

In order to solve system (36), the nodal vectors are subdivided into known and unknown parts denoted by the superscripts $k$ and $u$.

$$
\left\{U^{k}, T^{u}\right\} \in \Gamma_{u}, \quad\left\{U^{u}, T^{k}\right\} \in \Gamma_{T}
$$

The following matrix equation is obtained from Eq. (36). 


$$
\left[\begin{array}{ll}
M^{11} & M^{12} \\
M^{21} & M^{22}
\end{array}\right]\left[\begin{array}{l}
\ddot{U}^{k} \\
\ddot{U}^{u}
\end{array}\right]+\left[\begin{array}{ll}
K^{11} & K^{12} \\
K^{21} & K^{22}
\end{array}\right]\left[\begin{array}{l}
U^{k}(t) \\
U^{u}(t)
\end{array}\right]=\left[\begin{array}{ll}
\eta^{11} & \eta^{12} \\
\eta^{21} & \eta^{22}
\end{array}\right]\left[\begin{array}{l}
T^{k}(t) \\
T^{u}(t)
\end{array}\right]+\left[\begin{array}{l}
\mathcal{B}^{1}(t) \\
\mathcal{B}^{2}(t)
\end{array}\right]
$$

The unknown fluxes $T^{u}(t)$ are obtained from the first row of matrix Eq. (24) and are expressed as follows.

$$
\begin{aligned}
T^{u}(t)=( & \left.\eta^{12}\right)^{-1}\left[M^{11} \ddot{U}^{k}(t)+M^{12} \ddot{U}^{u}(t)+K^{11} U^{k}(t)\right. \\
& \left.+K^{11} U^{k}(t)+K^{12} U^{u}(t)-\eta^{11} T^{k}(t)-\mathcal{B}^{1}(t)\right]
\end{aligned}
$$

Making use of Eq. (40), we can write the second row of matrix Eq. (39) as follows

$$
M^{\mathrm{u}} \ddot{U}^{\mathrm{u}}(\mathrm{t})+\mathrm{K}^{\mathrm{u}} \mathrm{U}^{\mathrm{u}}(\mathrm{t})=\mathrm{Q}^{\mathrm{k}}(\mathrm{t})
$$

where

$$
\begin{aligned}
Q^{k}(t) & =\overline{\mathcal{B}}^{k}(t)+\eta^{k} T^{k}(t)-M^{k} \ddot{U}^{k}(t)-K^{k} U^{k}(t) \\
M^{u} & =M^{22}-\eta^{22}\left(\eta^{12}\right)^{-1} M^{12} \\
M^{k} & =M^{21}-\eta^{22}\left(\eta^{12}\right)^{-1} M^{11} \\
K^{u} & =K^{22}-\eta^{22}\left(\eta^{12}\right)^{-1} K^{12} \\
K^{k} & =K^{21}-\eta^{22}\left(\eta^{12}\right)^{-1} K^{11} \\
\eta^{k} & =\eta^{21}-\eta^{22}\left(\eta^{12}\right)^{-1} \eta^{11} \\
\overline{\mathcal{B}}^{k}(t) & =\mathcal{B}^{2}(t)-\eta^{22}\left(\eta^{12}\right)^{-1} \mathcal{B}^{1}(t)
\end{aligned}
$$

We now split the system (41) into elastic and electric parts as follows:

$$
\left[\begin{array}{ll}
M_{u u}^{u} & 0 \\
M_{\varphi u}^{u} & 0
\end{array}\right]\left[\begin{array}{c}
\ddot{u}^{u}(t) \\
\ddot{\varphi}^{u}(t)
\end{array}\right]+\left[\begin{array}{ll}
K_{u u}^{u} & K_{u \varphi}^{u} \\
K_{\varphi u}^{u} & K_{\varphi \varphi}^{u}
\end{array}\right]\left[\begin{array}{c}
u^{u}(t) \\
\varphi^{u}(t)
\end{array}\right]=\left[\begin{array}{c}
Q_{u}^{k}(t) \\
Q_{\varphi}^{k}(t)
\end{array}\right]
$$

The unknown electric potential $\varphi^{\mathrm{u}}$ can be obtained from the second row of Eq. (42) as

$$
\varphi^{u}(t)=\left(K_{\varphi \varphi}^{u}\right)^{-1}\left[Q_{\varphi}^{k}(t)-M_{\varphi u}^{u} \ddot{u}^{u}(t)-K_{\varphi u}^{u} u^{u}(t)\right]
$$

With the use of Eq. (43) into the first row of Eq. (42) we obtain

$$
\bar{M}^{u} \ddot{u}^{u}(t)+\bar{K}^{u} u^{u}(t)=\bar{Q}^{k}(t)
$$

Where 


$$
\begin{aligned}
\bar{Q}^{k}(t) & =Q_{u}^{k}(t)-K_{u \varphi}^{u}\left(K_{\varphi \varphi}^{u}\right)^{-1} Q_{\varphi}^{k}(t) \\
\bar{M}^{u} & =M_{u u}^{u}-K_{u \varphi}^{u}\left(K_{\varphi \varphi}^{u}\right)^{-1} M_{\varphi u}^{u} \\
\bar{K}^{u} & =K_{u u}^{u}-K_{u \varphi}^{u}\left(K_{\varphi \varphi}^{u}\right)^{-1} K_{\varphi u}^{u}
\end{aligned}
$$

Now, writing Eq. (44) for the $\mathrm{n}+1$ th time step

$$
\bar{M}^{u} \ddot{u}_{n+1}^{u}+\bar{K}^{u} u_{n+1}^{u}=\bar{Q}_{n+1}^{k}
$$

where

$$
\bar{Q}_{n+1}^{k}=\overline{\mathcal{B}}_{n+1}^{k}+\eta^{k} T_{n+1}^{k}-M^{k} \ddot{u}_{n+1}^{k}-K^{k} u_{n+1}^{k}
$$

The Newmark time integration algorithm was used to reduce the system of ordinary differential Eq. (45) to an algebraic system. The displacements $u_{n+1}$ and velocities $\dot{u}_{n+1}$ used in this algorithm are approximated at time step $n+1$ as follows:

$$
\begin{gathered}
\dot{u}_{n+1} \approx \dot{u}_{n}+\left[(1-\delta) \ddot{u}_{n}+\delta \ddot{u}_{n+1}\right] \Delta t \\
u_{n+1} \approx u_{n}+\dot{u}_{n} \Delta t+\left[\left(\frac{1}{2}-\alpha\right) \ddot{u}_{n}+\alpha \ddot{u}_{n+1}\right] \Delta t^{2}
\end{gathered}
$$

The acceleration at time step $n+1$ may be expressed from Eq. (48) as:

$$
\ddot{u}_{n+1} \approx \frac{1}{\alpha \Delta t^{2}}\left(u_{n+1}-u_{n}\right)-\frac{1}{\alpha \Delta t} \dot{u}_{n}-\left(\frac{1}{2 \alpha}-1\right) \ddot{u}_{n}
$$

Upon substitution of (49) into (45) we obtain the following algebraic system

$$
\mathbb{R} u_{n+1}^{u}=\mathcal{M}_{n+1}
$$

where the stiffness matrix $\mathbb{R}$ and effective load vector $\mathcal{M}_{n+1}$ are given by

$$
\begin{gathered}
\mathbb{R}=\frac{1}{\alpha \Delta t^{2}} \bar{M}^{u}+\bar{K}^{u} \\
\mathcal{M}_{n+1}=\bar{Q}_{n+1}^{k}+\bar{M}^{u}\left[\frac{1}{\alpha \Delta t^{2}} u_{n}^{u}+\frac{1}{\alpha \Delta t} \dot{u}_{n}^{u}+\left(\frac{1}{2 \alpha}-1\right) \ddot{u}_{n}^{u}\right]
\end{gathered}
$$

Once we have solved (50) for the unknown displacements at time step $n+1$, we can compute the accelerations and velocities from Eqs. (49) and (47) respectively. Finally, the electric potential $\varphi^{u}(t)$ can be obtained from (43) and the unknown generalized tractions $T^{u}(t)$ can be determined using Eq. (40). 


\section{Numerical Results and Discussion}

With the view of illustrating the numerical results calculated by method presented in this paper, the material chosen for the plate is the piezoelectric ceramic Lead Zirconate Titanate (PZT), and the physical data for which is given as follows:

The elasticity tensor $C$, piezoelectric tensor $e$ and relative permittivity $\epsilon^{\text {rel }}$

$$
\begin{aligned}
C_{f g h i}= & \left(\begin{array}{cccccc}
107.6 & 63.10 & 63.90 & 0.000 & 0.000 & 0.000 \\
63.10 & 107.6 & 63.90 & 0.000 & 0.000 & 0.000 \\
63.90 & 63.90 & 100.4 & 0.000 & 0.000 & 0.000 \\
0.000 & 0.000 & 0.000 & 19.60 & 0.000 & 0.000 \\
0.000 & 0.000 & 0.000 & 0.000 & 19.60 & 0.000 \\
0.000 & 0.000 & 0.000 & 0.000 & 0.000 & 22.20
\end{array}\right) \\
e & =\left(\begin{array}{cccccc}
0.00 & 0.00 & 0.00 & 0.00 & 12.0 & 0.00 \\
0.00 & 0.00 & 0.00 & 12.0 & 0.00 & 0.00 \\
-9.6 & -9.6 & 15.1 & 0.00 & 0.00 & 0.00
\end{array}\right) \\
\epsilon^{r e l}=\left(\begin{array}{cccc}
1936 & 0.00 & 0.00 \\
0.00 & 1936 & 0.00 \\
0.00 & 0.00 & 2109
\end{array}\right) &
\end{aligned}
$$

Mass density $\rho=7820 \mathrm{~kg} / \mathrm{m}^{3}$ and $\Delta \tau=0.0001$.

In order to evaluate the influence of the gravitational waves for HPS and FGPS on the displacements, for FGPS the inhomogeneity parameter is taken to be $m=0.5$ and for HPS case, we assume that $m=0$.

Figures 2, 3 and 4 describe anisotropic displacement behaviour of the HPS and FGPS under the influence of gravitational waves. A comparison of the results is presented graphically for the following different cases: the solid line denoted by " $\mathrm{A}$ " represents the solution for FGPS in the absence of gravitational waves $(g=0)$, the dashed line denoted by "B" represents the solution for HPS in the presence of gravitational waves $(\mathrm{g}=9.8)$, the dotted line denoted by " $\mathrm{C}$ " represents the solution for FGPS in the presence of gravitational waves $(\mathrm{g}=9.8)$ and the dashed-dotted line denoted by " $D$ " represents the solution for HPS in the absence of gravitational waves $(\mathrm{g}=0)$.

Figure 2 shows that the displacement $u_{1}$ increases with $\mathrm{x}$ for all cases.

Figure 3 shows that the displacement $u_{2}$ increases with $\mathrm{x}$ then it decreases with $\mathrm{x}$ until $\mathrm{x}=0.085$ for FGPS under the effect of gravitational waves, but for HPS it increases with $\mathrm{x}$.

Figure 4 shows that the displacement $u_{3}$ decreases with increasing $\mathrm{x}$ for FGPS until $\mathrm{x}=0.45$ then it increases with increasing $\mathrm{x}$. Also, it decreases with increasing $\mathrm{x}$ for HPS until $x=0.2$ then it increases with increasing $x$.

Since the gravitational waves act in the $z$-direction; the great effect of it may be very clearly in the $u_{3}$ component. 


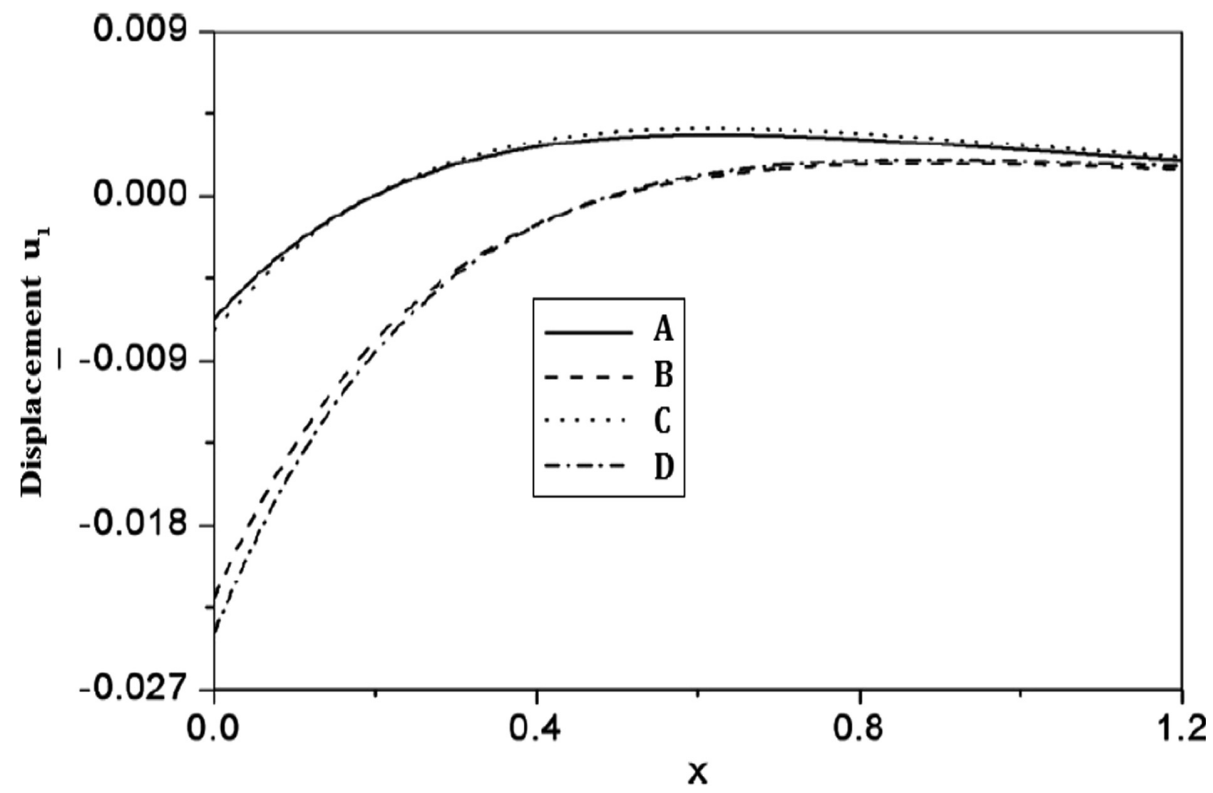

Fig. 2. Displacement distribution under four cases.

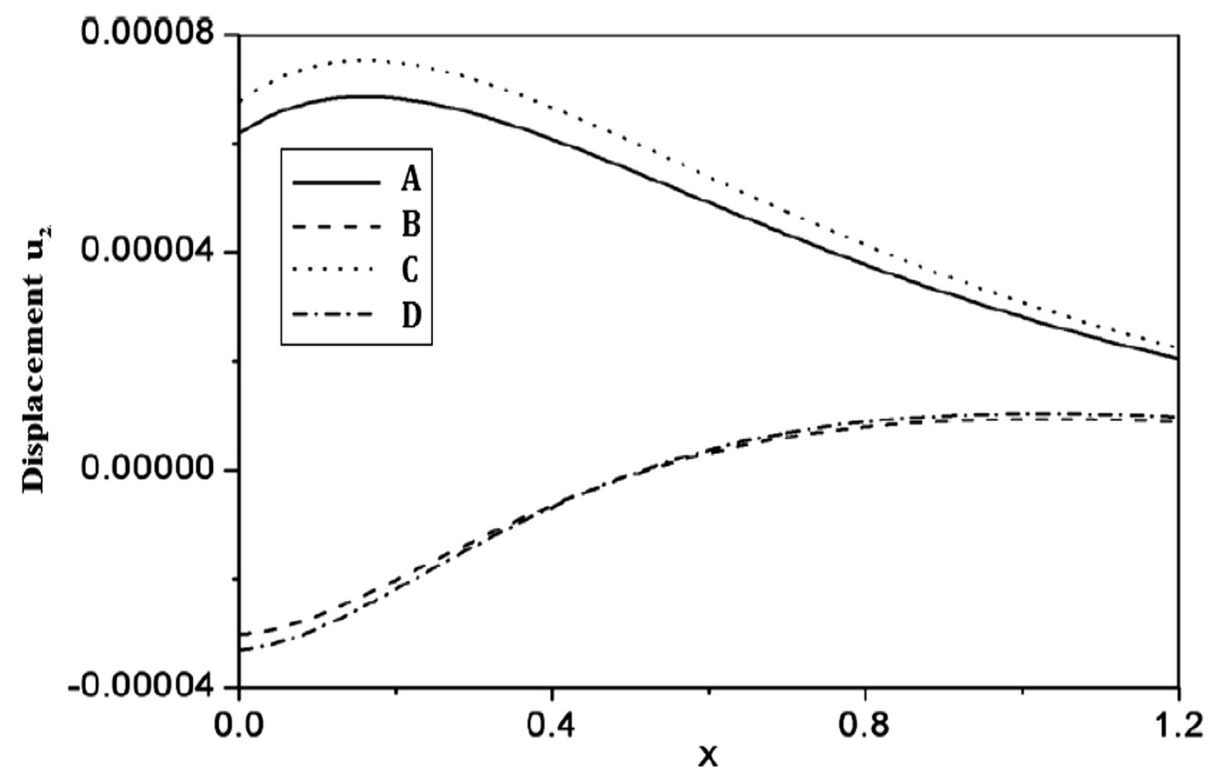

Fig. 3. Displacement distribution under four cases. 


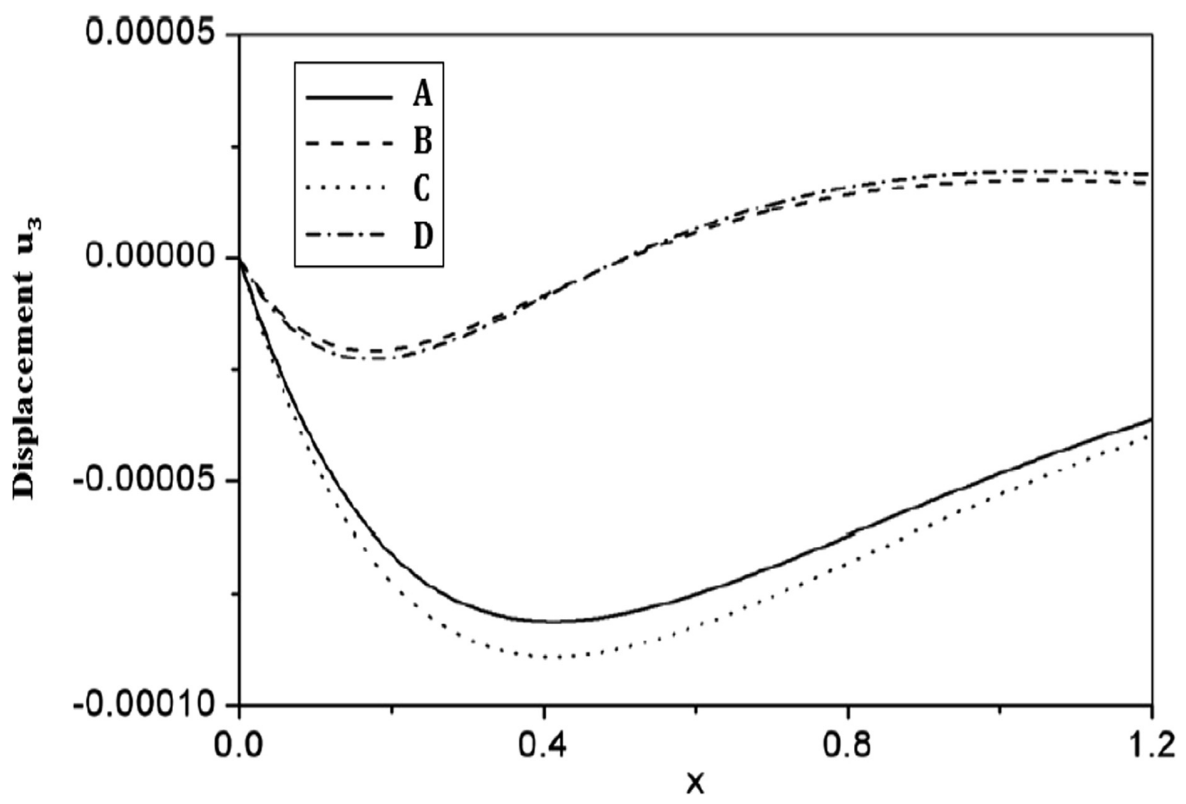

Fig. 4. Displacement distribution under four cases.

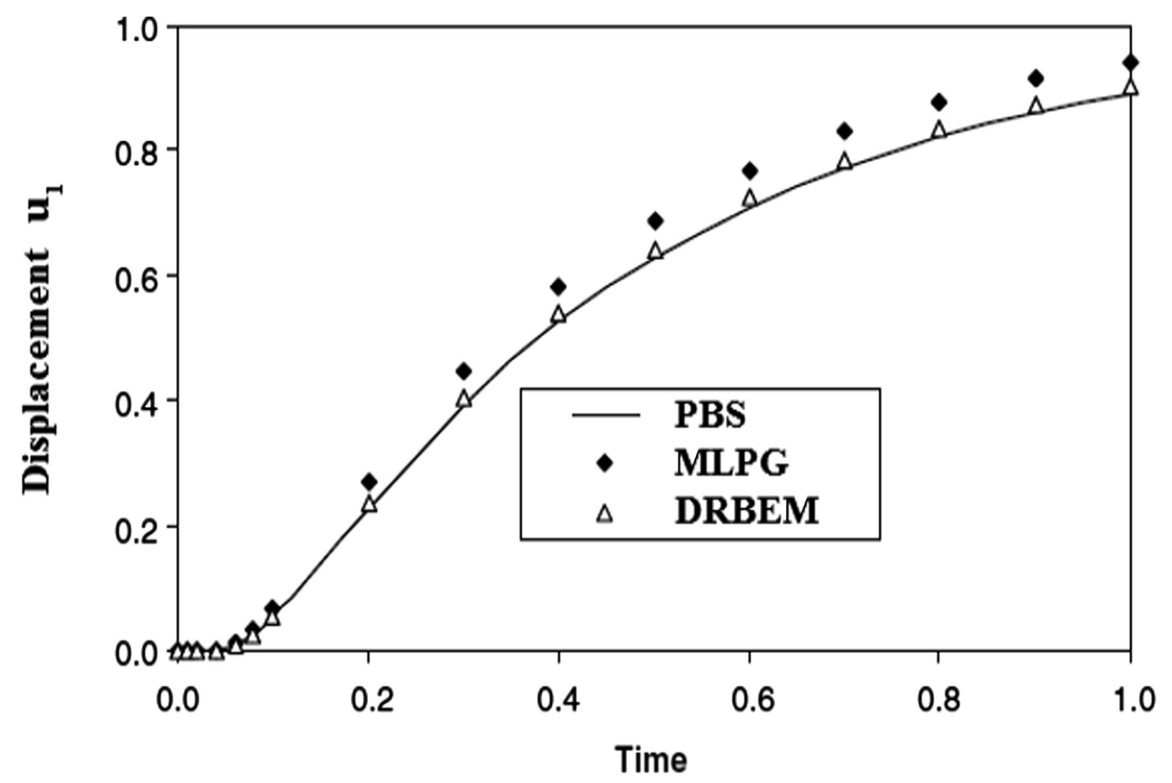

Fig. 5. Variation of the Displacement $\mathrm{u}_{1}$ with time for three methods. 


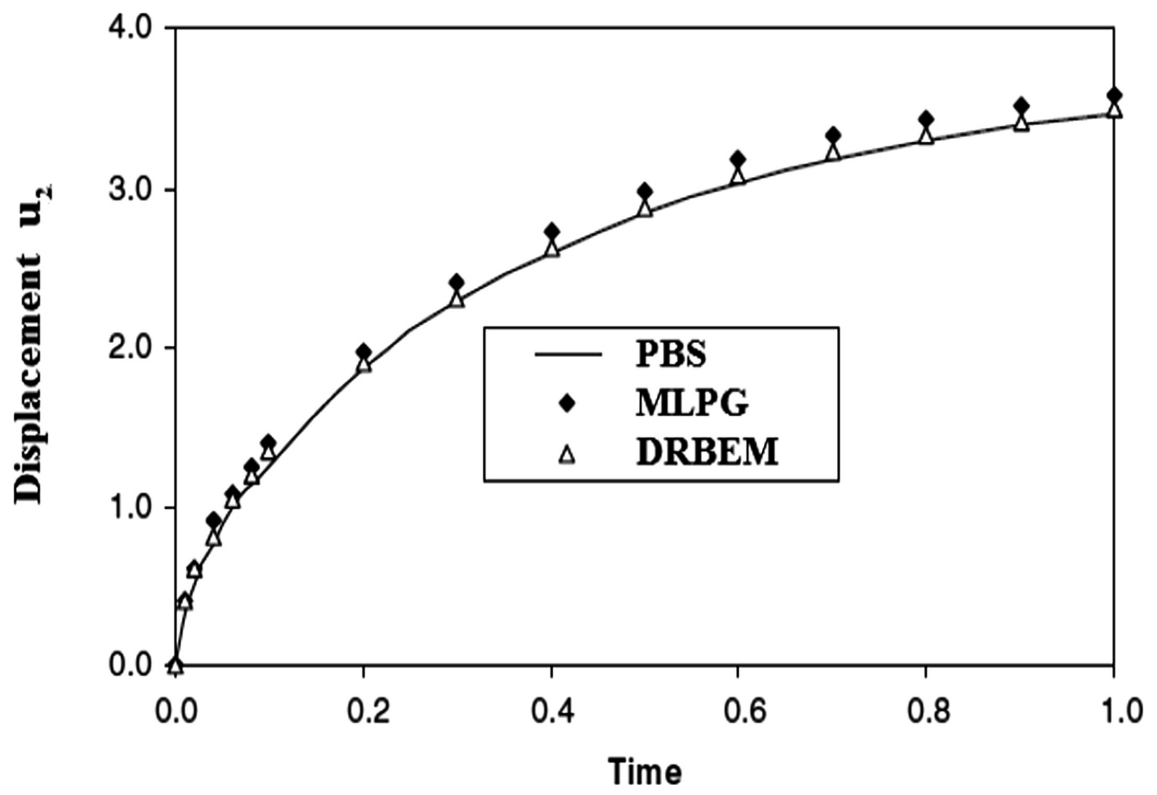

Fig. 6. Variation of the displacement $\mathrm{u}_{2}$ with time for three methods.

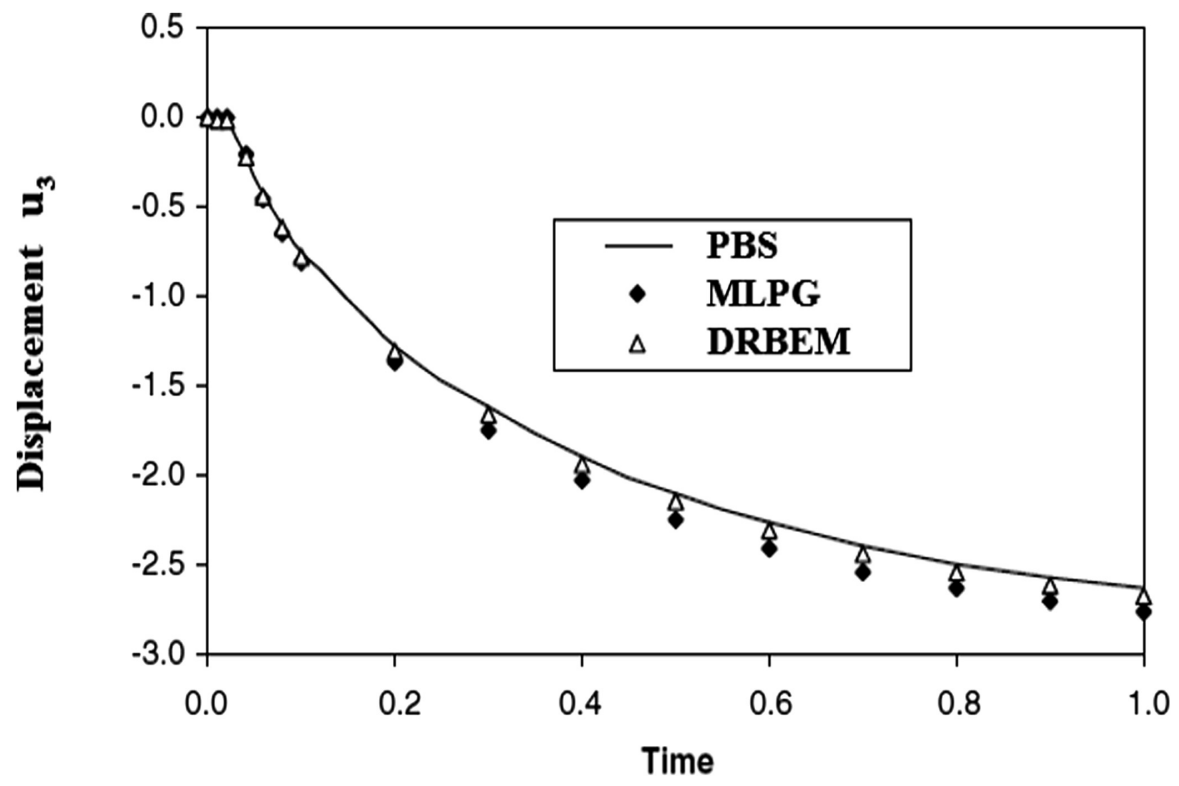

Fig. 7. Variation of the displacement $u_{3}$ with time for three methods. 
It is noticed from these figures that the effect of gravitational waves is weak, where its effect cannot be neglected but the functionally graded effect is strong, and the functionally graded effect is more clear in the results obtained at $\mathrm{u}_{2}$.

In the special case under consideration, the results are plotted in Figs. 5, 6 and 7 to show the validity of the DRBEM. These results obtained with the DRBEM have been compared graphically with those obtained using the Meshless Local Petrov-Galerkin (MLPG) method of (Stanak et al. 2013) and the Peano-Baker Series (PBS) method of (Liu et al. 2014). It can be seen from these figures that the DRBEM results are in excellent agreement with the results obtained by MLPG and PBS methods, thus confirming the accuracy of the DRBEM.

\section{Conclusions}

A three dimensional time-stepping dual reciprocity boundary element method (DRBEM) modelling was investigated for anisotropic HPS and FGPS under the influence of gravitational waves. The FGPS is assumed to be graded through the thickness. This paper investigated the effects of gravitational waves on the HPS and FGPS. The accuracy of the proposed method was examined and confirmed by comparing the obtained results with those known previously, and excellent agreement is obtained with the results obtained by other methods. Understanding the behaviour of anisotropic HPS and FGPS under the effect of gravitational waves should be a key for extending the application of these structures.

\section{References}

Abd-Alla, A.N., Alsheikh, F.A.: Reflection and refraction of plane quasi-longitudinal waves at an interface of two piezoelectric media under initial stresses. In: Archive of Applied Mechanics. Springer (2009a). doi:10.1007/s00419-008-0257-y

Abd-Alla, A.N., Al-sheikh, F.A.: The effect of the initial stresses on the reflection and transmission of plane quasi-vertical transverse waves in piezoelectric materials. World Acad. Sci. Eng. Technol. 50, 660-668 (2009)

Abd-Alla, A.N., et al.: The reflection phenomena of quasi-vertical transverse waves in piezoelectric medium under initial stresses. In: Meccanica. Springer (2012). doi:10.1007/ s11012-011-9485-2

Alibeigloo, A., Liew, K.M.: Elasticity solution of free vibration and bending behavior of functionally graded carbon nanotube-reinforced composite beam with thin piezoelectric layers using differential quadrature method. Int. J. Appl. Mech. World Sci. 7(1), 1550002 (2015). doi:10.1142/S1758825115400025

Davì, G., Milazzo, A.: A regular variational boundary model for free vibrations of magneto-electro-elastic structures. Eng. Anal. Boundary Elem. (2011). doi:10.1016/j. enganabound.2010.10.004. Elsevier

Fahmy, M.A.: A time-stepping DRBEM for magneto-thermo-viscoelastic interactions in a rotating nonhomogeneous anisotropic solid. Int. J. Appl. Mech. (2011). doi:10.1142/ S1758825111001202. Imperial College Press 
Fahmy, M.A.: A time-stepping DRBEM for the transient magneto-thermo-visco-elastic stresses in a rotating non-homogeneous anisotropic solid. Eng. Anal. Boundary Elem. (2012a). doi:10. 1016/j.enganabound.2011.09.004. Elsevier

Fahmy, M.A.: Numerical modeling of transient magneto-thermo-viscoelastic waves in a rotating nonhomogeneous anisotropic solid under initial stress. Int. J. Model. Simul. Sci. Comput. (2012b). doi:10.1142/S179396231250002X. World Scientific

Fahmy, M.A.: Transient magneto-thermo-elastic stresses in an anisotropic viscoelastic solid with and without moving heat source. Numer. Heat Transf. Part A: Appl. (2012). doi:10.1080/ 10407782.2012.667322. Taylor Francis

Fahmy, M.A.: Transient magneto-thermoviscoelastic plane waves in a non-homogeneous anisotropic thick strip subjected to a moving heat source. Appl. Math. Modell. (2012c). doi:10.1016/j.apm.2011.11.036. Elsevier

Fahmy, M.A.: Transient magneto-thermo-viscoelastic stresses in a rotating nonhomogeneous anisotropic solid with and without a moving heat source. J. Eng. Phys. Thermophys. (2012d). doi:10.1007/s10891-012-0735-5. Springer

Fahmy, M.A.: The effect of rotation and inhomogeneity on the transient magneto thermoviscoelastic stresses in an anisotropic solid. J. Appl. Mech. ASME 10(1115/1), 4006258 (2012f). doi:10.1115/1.4006258

Fahmy, M.A.: Generalized magneto-thermo-viscoelastic problems of rotating functionally graded anisotropic plates by the dual reciprocity boundary element method. J. Thermal Stress (2013a). doi:10.1080/01495739.2013.765206. Taylor and Francis

Fahmy, M.A.: A three-dimensional generalized magneto-thermo viscoelastic problem of a rotating functionally graded anisotropic solids with and without energy dissipation. Numer. Heat Transf. Part A: Appl. (2013). doi:10.1080/10407782.2013.751317. Taylor and Francis

Fahmy, M.A.: Implicit-explicit time integration DRBEM for generalized magneto-thermoelasticity problems of rotating anisotropic viscoelastic functionally graded solids. Eng. Anal. Boundary Elem. (2013b). doi:10.1016/j.enganabound.2012.08.002

Fahmy, M.A.: A computerized DRBEM model for generalized magneto-thermo-visco-elastic stress waves in functionally graded anisotropic thin film/substrate structures. Latin Am. J. Solids Struct. Scielo (2014). doi:10.1590/S1679-78252014000300003

Fahmy, M.A.: Computer implementation of the DRBEM for studying the generalized thermo elastic responses of functionally graded anisotropic rotating plates with two relaxation times. Br. J. Math. Comput. Sci. (2014b). doi:10.9734/BJMCS/2014/7391. Sciencedomain International

Fang, D.N., et al.: Magnetoelastic fracture of soft ferromagnetic materials. Theoret. Appl. Fracture Mech. (2004). doi:10.1016/j.tafmec.2004.09.006. Elsevier

Frijns, J.H., et al.: Improving the accuracy of the boundary element method by the use of second-order interpolation functions. IEEE (2000). doi:10.1109/10.871407. http://www.ncbi. nlm.nih.gov/pubmed/11059168

Gaul, L., et al.: Boundary element methods for engineers and scientists. Springer (2003). doi:10. 1007/978-3-662-05136-8

Jin, B., Zhong, Z.: A moving mode-III crack in functionally graded piezoelectric material: permeable problem. Mech. Res. Commun. (2002). doi:10.1016/S0093-6413(02)00259-8. Elsevier

Kuna, M.: Fracture mechanics of piezoelectric materials - where are we right now? Eng. Fracture Mech. (2010). doi:10.1016/j.engfracmech.2009.03.016. Elsevier

Lin, S., et al.: Electroelastic analysis of a penny-shaped crack in a piezoelectric ceramic under mode I loading. Mech. Res. Commun. (2003). doi:10.1016/S0093-6413(03)00031-4. Elsevier 
Liu, W., et al.: Three-dimensional analysis of functionally graded piezoelectric plate with arbitrarily distributed material properties. J. Wuhan Univ. Technol.-Mater. Sci. (2014). doi:10.1007/s11595-014-0985-5. Springer

Nardini, D., Brebbia, C.A.: A new approach to free vibration analysis using boundary elements. Appl. Math. Modell. (1983). doi:10.1016/0307-904X(83)90003-3. Elsevier

Pan, E.A.: BEM analysis of fracture mechanics in 2D anisotropic piezoelectric solids. Eng. Anal. Boundary Elem. (1999). doi:10.1016/S0955-7997(98)00062-9. Elsevier

Partridge, P.W., Brebbia, C.A.: Computer implementation of the BEM dual reciprocity method for the solution of general field equations. Commun. Appl. Numer. Methods (1990). doi:10. 1002/cnm.1630060204. Wiley

Partridge, P.W., Wrobel, L.C.: The dual reciprocity boundary element method for spontaneous ignition. Int. J. Numer. Methods Eng. (1990). doi:10.1002/nme.1620300502. Wiley

Stanak, P., et al.: Three-dimensional meshless modelling of functionally graded piezoelectric sensor. In: Mechatronics 2013. Springer (2014). doi:10.1007/978-3-319-02294-9_54

Suo, Z., et al.: Fracture mechanics for piezoelectric ceramics. J. Mech. Phys. Solids (1992). doi:10.1016/0022-5096(92)90002-J. Elsevier

Wrobel, L.C., Brebbia, C.A.: The dual reciprocity boundary element formulation for nonlinear diffusion problems. Comput. Methods Appl. Mech. Eng. (1987). doi:10.1016/0045-7825(87) 90010-7. Elsevier

Zhang, T.Y., et al.: Fracture of piezoelectric ceramics. Adv. Appl. Mech. (2002). doi:10.1016/ S0065-2156(02)80104-1. Elsevier 